\title{
Measuring the performance of railway track through large scale trackside sensor deployments
}

\author{
David Milne ${ }^{1[0000-0001-6702-3918]}$, Louis Le Pen ${ }^{1[0000-0002-4362-3895]}$, Geoff Watson ${ }^{1[0000-0003-}$ \\ 3074-5196] and William Powrie ${ }^{[0000-0002-2271-0826]}$ \\ ${ }^{1}$ University of Southampton, Southampton, UK \\ d.milne@soton.ac.uk
}

\begin{abstract}
Railway track performance is inherently variable along its length, even on straight plain line track. Current condition monitoring relies on snapshot train borne measurements of track geometry, which may be used to plan route level maintenance. Although this provides a global record of current track quality, it is difficult to infer the local condition of the trackbed and local mechanisms of deterioration that may be occurring. A more important indicator of local trackbed condition may be the trackbed support stiffness as seen by a train. The trackbed support stiffness influences the performance of the track and its variation could potentially be used to predict changes in longer term performance with trafficking by means of vehicle / track interaction models and settlement equations. Trackside measurements may be obtained using track mounted sensors such as accelerometers, geophones, deflectometers, high speed video cameras or strain gauges. These may be interpreted to determine the trackbed support stiffness. However, many measurement locations are required to determine the variation in trackbed stiffness along a useful length of track. This requires a significant increase in the scale of typical trackside monitoring deployments, potentially generating large volumes of data and requiring a degree of automation for data processing. The availability of small, inexpensive, standalone micro electromechanical systems (MEMS) accelerometers means that it is now practicable to instrument hundreds of sleeper-ends in a single deployment, covering far greater lengths of track than would be viable with more established and expensive trackside monitoring approaches. This paper shows how MEMS have been used to investigate the performance of longer sections of track. Data processing techniques are described, and insights into the actual variation of track system performance are discussed.
\end{abstract}

Keywords: Rail track substructures, including transition zones, and transportation geodynamics, accelerometers, ties, sleepers, trackbed stiffness, support modulus. 


\section{Introduction}

Route level maintenance of railway tracks is generally based on train borne measurements of track geometry [1]. Geometry parameters such as level or alignment are relative measures in that they describe the deviation from the average over a particular wavelength, rather than the absolute position of the track. Infrastructure managers use these data to plan track-bed maintenance, targeting both significant deviations in local geometry and high variability over fixed lengths of track. The implication of this is that differential settlement of the track will be a driver for track-bed maintenance. If differential behavior is important, variations in the track performance, loading (from vehicle track interaction) and track properties (stiffness) along the length of the track need to be understood. Taking an evidenced based, data rich approach to studying this will require large scale monitoring with resolution at a local scale.

One approach for investigating the performance of railway track is lineside monitoring, in which different sensors placed at intervals along the track superstructure and used to record the response of the track under traffic e.g. [2-6]. Typical lineside deployments tend to be small and highly localized owing to the costs of the instrumentation and analysis requirements. To investigate variability over longer lengths of track, the instrumentation, data acquisition and data processing / interpretation requirements will increase significantly. One implication of this is that lower cost instrumentation is needed to enable larger scale deployments; another is that data processing and basic interpretation will need to be automated to study the variability of the track from the trackside measurements. The same would likely be required for any long term, pervasive monitoring of critical parts of the infrastructure.

This paper explores how large numbers of comparatively low-cost MEMS accelerometers can be deployed and the data obtained processed to provide insights into the variability of railway track performance. Criteria for the selection of accelerometers are discussed, processing techniques described, and their application demonstrated.

\section{Methods}

\subsection{Sensors}

One of the most common lineside monitoring approaches is the use of inertial sensors to record track vibration. Displacement can be obtained by filtering then integrating once for velocity or twice for acceleration measurements [7]. Application of a high pass filter, necessary to avoid low frequency drift when integrating, results in startup transients and a shift in the apparent datum for the deflection data; a statistical method for correcting for this and assessing the range of deflection is described later.

Micro electro mechanical systems (MEMS) accelerometers, often used in consumer electronics, are far less expensive than more conventional research-quality devices (costing tens rather than hundreds of dollars), potentially enabling much larger deployments. 
Deflection of railway track is a low frequency phenomenon [8]. This has implications for the selection of monitoring accelerometers, as the accelerations significant for deflection are likely to be of low amplitude and low frequency. Several facets of performance for both the transducer and the data acquisition system need to be considered, depending on where within the track system a sensor is to be deployed. For, example a sensor on the rail will experience higher amplitude, higher frequency vibration than a sensor on a sleeper or in the track bed.

- Accuracy is limited by noise, as this affects the minimum acceleration that will be detectable at a given frequency, how accurately a change in acceleration can be resolved and whether acceleration data are likely to integrate to produce meaningful deflections. This is important in the current application because amplification of transducer noise is inversely proportional to the square of frequency of interest.

- Range and resolution - the range defines amplitude of acceleration over which a sensor can operate without saturating. The voltage range and / or bit depth over which this is discretized defines the precision of the monitoring system.

- Sample rate is controlled by which frequencies are of interest and the sensor bandwidth, i.e. the frequency range that a sensor can detect. The sensor should be able to detect all frequencies of interest and the data acquisition system must be capable of sample rates of twice the sensor bandwidth to satisfy the sampling theorem [9]. The bandwidth can be restricted by hard filtering the transducer outputs before analogue to digital conversion. Oversampling or similar signal conditioning can improve the effective bit depth or reduce noise.

Fairly inexpensive MEMS sensors have been shown capable of obtaining sleeper deflection from acceleration data, particularly at higher train speeds, producing reliable data for serval months on end [10]. At higher train speeds the frequencies and amplitudes of the acceleration signals are increased, improving the potential to detect them above sensor / system noise. MEMS sensors are available in variants that output both analogue and digital signals. Analogue sensor outputs require external analogue digital conversion (ADC) for datalogging, whereas digital variants include this within the sensor's integrated circuit (IC). Digital sensors require a microcontroller to access, transfer and store the data; these can be combined at board level, giving low cost standalone monitoring devices suited for monitoring infrastructure. An analogue sensor with an external ADC could, of course, be used with a microcontroller in a similar fashion.

The small size and IC form factor of MEMS sensors means that they can be assembled into a robust package. With appropriate power, communications systems / services and ruggedization these types of devices could form the basis of networked large-scale pervasive monitoring. This paper makes uses of a similar, but somewhat less sophisticated, standalone device, to demonstrate some potential applications of larger scale monitoring. The device used comprised an Analog Devices ADLX 345 triaxial MEMS accelerometer, microcontroller, real-time clock, micro SD card and lithium battery. This is a self-powered device, capable of sampling at $400 \mathrm{~Hz}$ (with $8 \times$ over sampling and finite impulse response (FIR) filtering) for later download to a computer via a USB. 
These devices were manufactured by Gulf Coast Data Concepts and cost around \$100. The accelerometer chip and measurement devices are shown in Fig. 1.

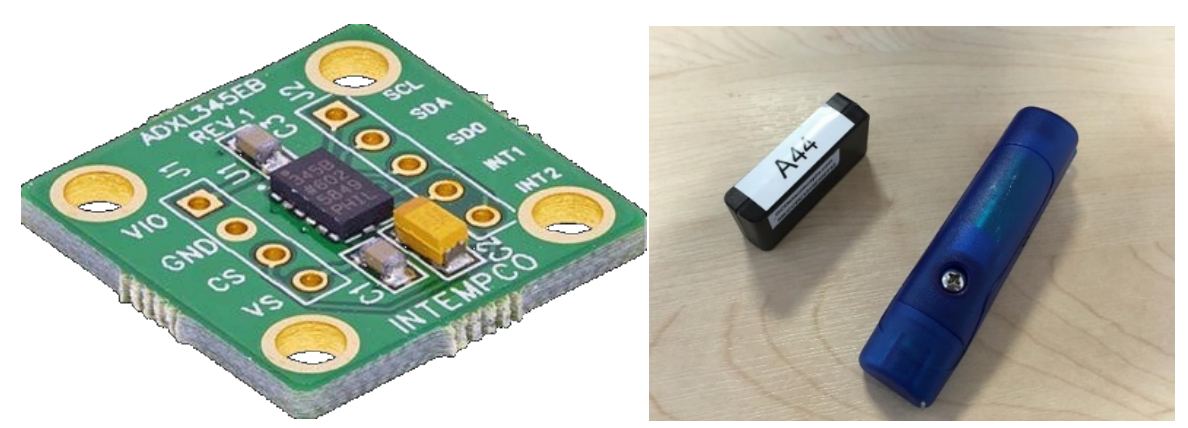

Fig. 1. MEMS accelerometer IC and standalone measurement devices (in two packaged equivalent variants)

Each device is independent and standalone; this means that in a deployment of multiple devices, measurements are simultaneous but not synchronous. Data are recorded continuously, as although the devices can be configured to start recording when a train is detected they do not buffer; thus, they cannot record the approach of the train, which is useful data for analysis.

\subsection{Data processing.}

In all the studies presented in this paper, the devices were placed on the track during a possession when no trains were able to pass. Devices were configured to start recording with the return of traffic, continuing until their batteries had depleted - usually between 4 and 8 hours. Sensors were then recovered during another possession. In some deployments, the recovered sensors were recharged and transferred to a new location. Data were then downloaded to a computer via a USB. These devices functioned reliably for these short but repeated deployments.

This approach generates large volumes of data, from which the valuable train passby data need to be extracted. The pass-by events can be extracted by retrospectively applying a threshold acceleration at a suitable level to identify when trains were present on the track. If the sensor clocks are approximately synchronized, the timestamp can be used to group, but not align, the same pass-by event recorded by different devices.

Analysis often requires the type of train to be identified, enabling like with like comparison and association of the trainset geometry and typical axle / wheel loads. This is challenging as the monitoring systems tend to be unattended. It may be done by inspection, using prior knowledge of which train types operate on the line. For pervasive monitoring, route data of train passing times could be linked into the analysis system. An approach yet to be explored is using machine learning to identify trains from the sensor records. 


\section{Deflection.}

A common objective of lineside monitoring is to assess the movement of railway track under load from trains. Once individual pass-by events have been identified and extracted, the data must be processed to obtain deflections. For acceleration data, this is done by filtering and integrating twice. Generally, a high pass filter is used to prevent low frequency drift during integration, and a low pass filter used to remove higher frequency noise and vibration not relevant to the major trackbed motions. The filter cut on / off frequencies must be selected so that the frequencies relevant for deflection are in the passband.

Fig. 2 shows acceleration data filtered and integrated to obtain deflection. The filtering affects the deflection trace obtained. There are transients at the start and end of the train passage and the data are averaged about zero, causing the apparent at rest position of the track to be shifted from zero.
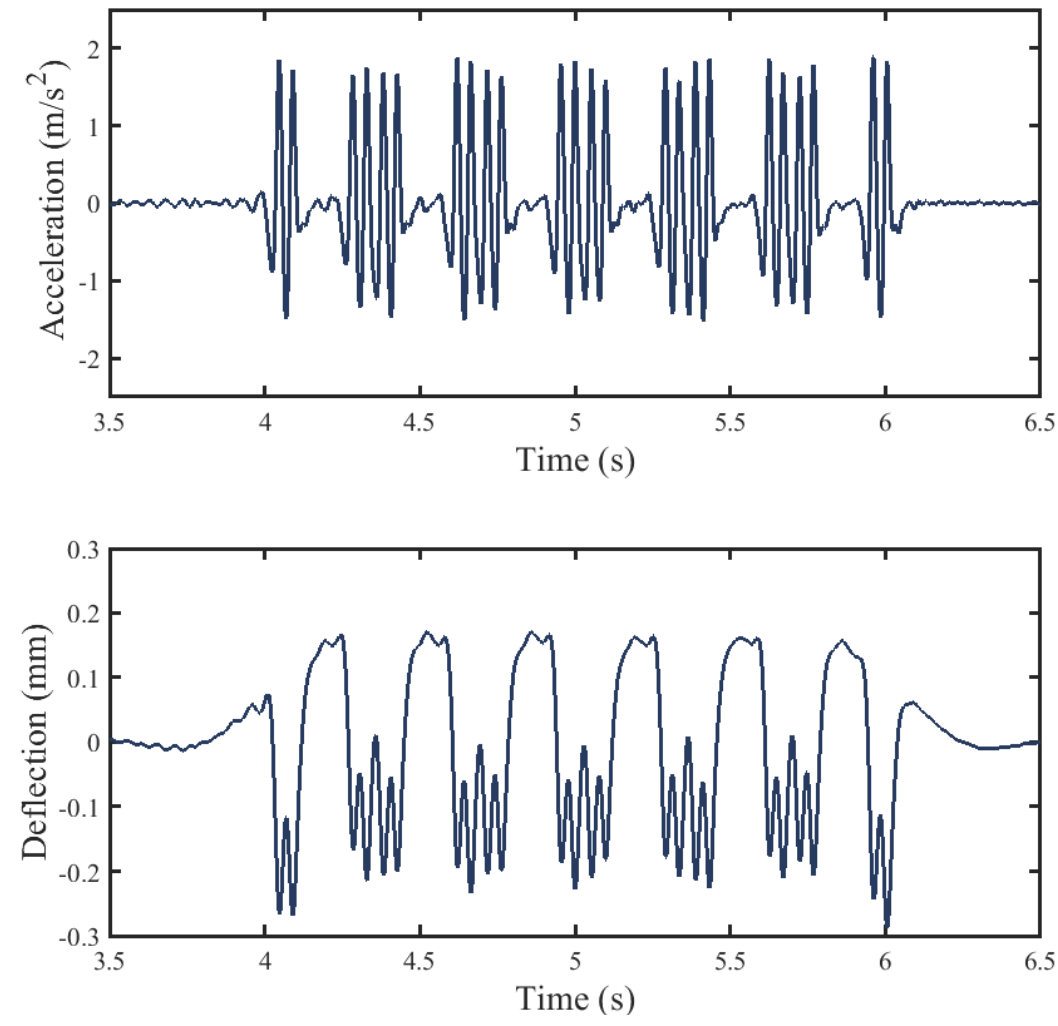

Fig. 2. Example acceleration signal integrated to obtain deflection 
If the data are of good quality and have been processed appropriately, there will be a "stationary" region containing a repeating pattern due to the passing train, visible for the four pairs of internal vehicle ends. [11] presents a statistical method using the cumulative distribution function for track deflection to calculate the proportion of the time that the track is expected to be above or below the rest position of the track. This can then be used to identify the at rest level of track and hence quantify the characteristic range of deflection, implicitly averaging over most of the train.

Fig. 3 shows the acceleration and deflection spectra for the data in Fig.2. The deflection spectrum shows that track deflection is a low frequency phenomenon, with the exact frequencies of interest dependent on the speed of the train. For passenger trains, repeating vehicles cause spectral peaks at multiples of the vehicle passing frequency (i.e., the length of the typical vehicle divided by the speed). The vehicle passing frequency can be considered to be the first frequency of interest for track deflection; the cut off frequency for the high pass filter should be between $1 / 2$ to $1 / 3$ of this frequency. [12] showed that including about 10 multiples of the vehicle passing frequency gives over $95 \%$ of the expected track deflection. The implication of this is that the cut-off on frequency for the low pass filter should be at least 10 multiples of the vehicle passing frequency to avoid filtering out information relevant for trackbed deflection.
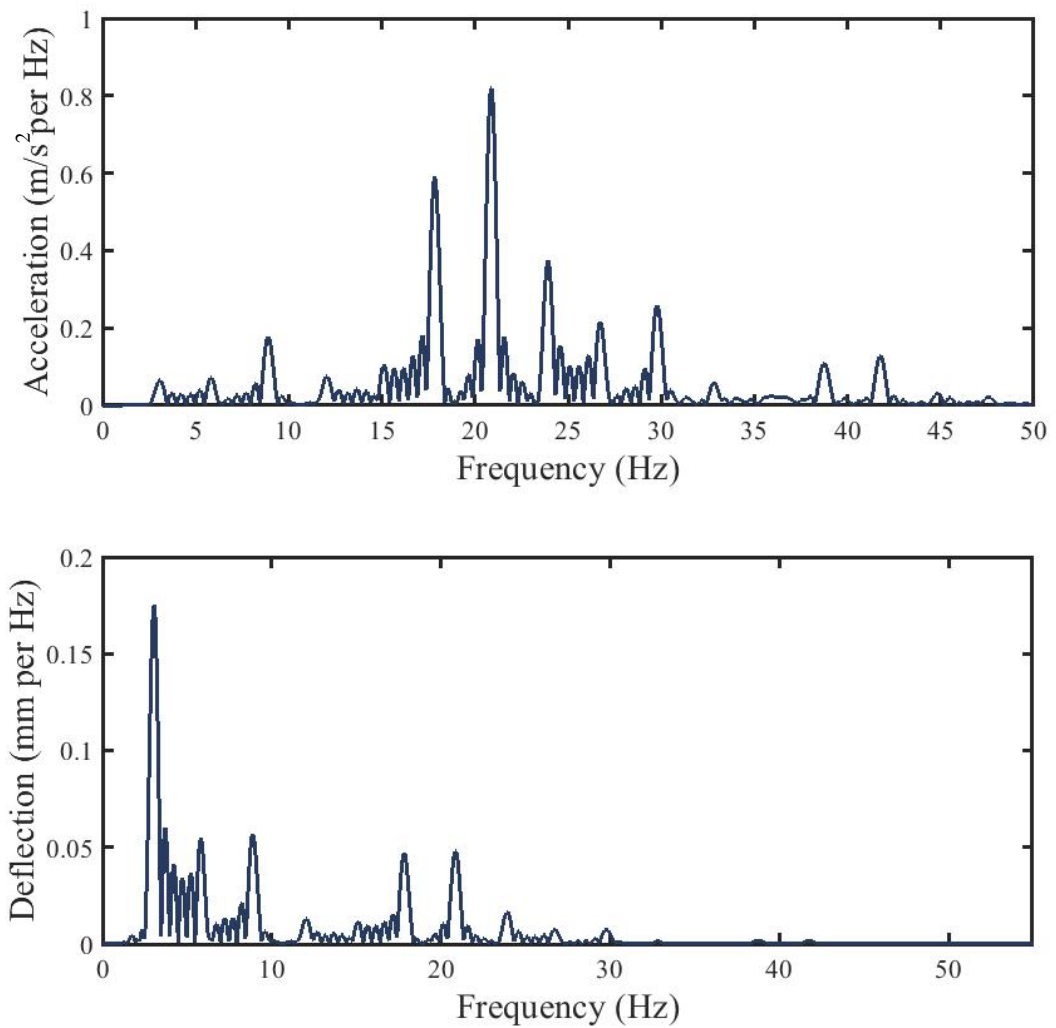
Fig. 3. Example acceleration and deflection spectra to $50 \mathrm{~Hz}$

\section{Stiffness.}

Another important performance indicator for different track systems is the stiffness, i.e. the amount it deflects for a given load. This may be defined in different ways, for example as a spring stiffness per sleeper end or as a linear modulus per metre of track. Determining a support stiffness or modulus requires knowledge of the applied load, the track deflection and a mathematical framework to account for the bending stiffness of the rails [13-15]. On a live railway, the applied load is both uncertain and challenging to measure. [16] proposed a method for determining the support system modulus by analysis in the frequency domain, without knowledge or measurement of the load. The dominant train load frequencies occur at multiples of the vehicle passing frequency [17], as may be seen in Fig. 3. The magnitudes of these frequencies depend on the train geometry and the track stiffness. [16] used the Fourier transform of the simple beam on an elastic foundation model for track deflection to develop a closed form solution relating the ratio of magnitudes of pairs of these dominant frequencies to the track system support modulus. Certain pairs of frequencies are better suited for this analysis than others [18].

\section{Along the track.}

The techniques described above enable characterization of individual train passage in terms of deflection and stiffness at discrete locations. Once categorized by train type, these data need to be interpreted into a profile showing how deflection and stiffness vary along the track. This is an interesting problem, with some similarities to time series (or trend) analysis.

In a deployment of a sensor array, multiple observations for the same train type should be made at each location, and ideally some average of these observations should be used to assess how the deflection and stiffness vary along the track. Variable train occupancy, speed and wheel condition will cause the results obtained for deflection and stiffness to vary from train to train at each location and there may be outliers. Owing to limitations on the time available, it may not be possible to make enough measurements to obtain a robust average and understand the distribution of deflection at a measurement point.

Hence the "best" (current) interpretation process for deflection and stiffness profiles is by a combination of curve fitting and inspection. In this process, every observation (for deflection or stiffness) for each sleeper is plotted along the track. A cubic 'not-aknot' spline is fitted to the data; this type of function suits rail deflection as it is continuous to its third derivative. Outliers are excluded by inspection, so that the curve is fitted to clusters of similar data at each measurement point. If enough observations are made, this stage is redundant. If data are missing or of low quality at some locations, curve fitting provides a way of estimating the likely performance, given the behavior 
of the surrounding track. A combination of clustering and curve fitting algorithms could be used to automate this process.

\section{$3 \quad$ Studies}

The techniques described above have recently been used to study the distribution of deflection and stiffness along different lengths of railway track in Europe [19, 20]. Two examples are presented here. These are a $200 \mathrm{~m}$ length of a ballasted high-speed railway track (site 1), with the aim of studying variation in performance; and a shorter length of track (site 2) where the techniques were used to measure the performance before and after a full trackbed renewal.

\subsection{Site 1}

Fig. 4 shows downward deflection and support system modulus data obtained over 350 sleepers on a ballasted high-speed railway. The results are based on acceleration measurements for the same type of six-vehicle passenger trains, which generally passed the site at a speed of $60 \mathrm{~m} / \mathrm{s}$. Results were obtained in three consecutive deployments in which the sensors were moved along the track during a night time possession. The results shown were averaged from data for between 6 and 12 train passes at each monitoring point.

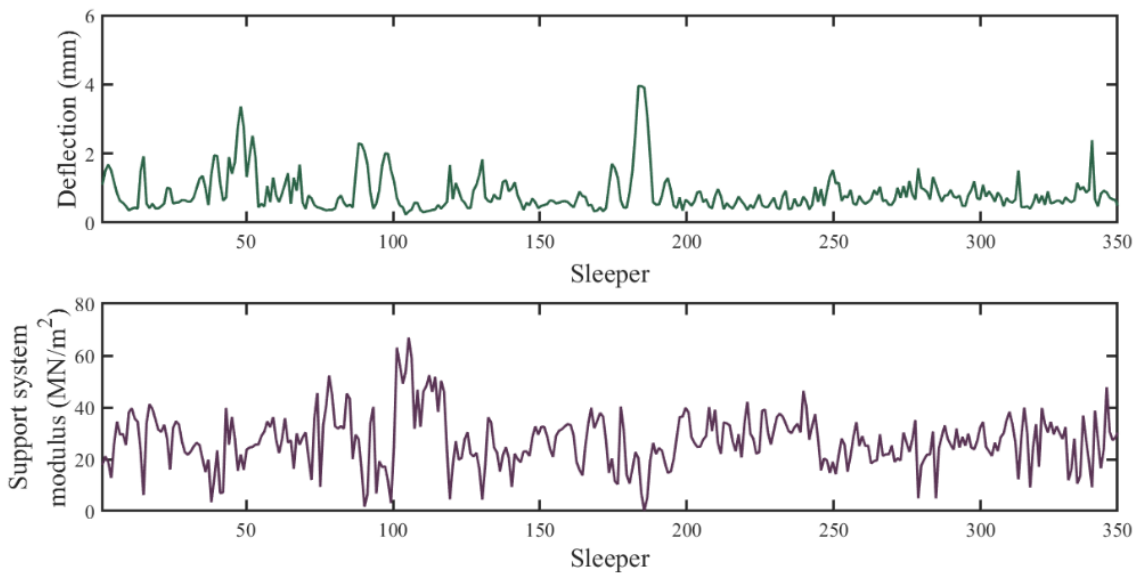

Fig. 4. Variation of deflection and support system modulus along $200 \mathrm{~m}$ of railway track [20].

The data in Fig. 4 provide insights into the real variation of deflection and stiffness along the track. Both measures vary from sleeper to sleeper, and areas of good and poor performance are clear. For example, the track deflections are more significant and variable between sleepers 0 and 200, with discrete zones of increased deflection around sleeper numbers 50, 90-100, 120-130 and 180, likely due to voiding between the sleeper 
and trackbed. The support system modulus is also more variable in this section. Both are indicative of poor performance. In contrast, from sleeper 200 onwards the deflections are smaller and more consistent, with a deflection floor of around $0.4 \mathrm{~mm}$ indicative of good performance. The support system modulus is also less variable for these sleepers. Discussion with local track engineers revealed that sleeper 200 marks approximately the end location of a tamping run, and that the section of the site up to this location had been tamped more frequently than the latter part. This has interesting implications, and further work could usefully look at whether the increased tamping frequency was causal or symptomatic.

In addition to allowing intuitive visualization of performance along a track, these data have been used by [19] to study, quantify and better understand the distribution of stiffness and deflection along a railway, providing a framework for both assessing and comparing variation in track conditions and potentially synthesizing realistic variations in track properties for simulation and forecasting. [20] used the data in combination with a track level survey and a simple vehicle-track interaction model to assess the significance of track level, track stiffness and geometric non-linearity from voiding for measured and simulated track performance. For this site, the permanent differences in track level were more significant for wheel rail contact forces than differences in support conditions. The support conditions, including non-linearity, were more important than initial level for determining track deflection.

\section{$3.2 \quad$ Site 2}

Fig. 5 shows track deflection data recently obtained using a deployment of about 40 accelerometers over a section of track running along an embankment, before and after a track renewal. Sleepers were replaced during the renewal, hence the sleeper numbers do not exactly correspond, and the data are not precisely aligned, although the two surveys are for the same section of track.

The data in Fig. 5 allow intuitive comparison of the condition of the track. Before the intervention, the performance was poor: deflections were large and variable (2-4 $\mathrm{mm}$ ), and there was a distinct fault with probable voiding. After the renewal, performance improved. All deflections were reduced to below $1.5 \mathrm{~mm}$. Sleepers 19-29 appear to be performing very well, achieving consistent performance with a deflection floor of around $0.6 \mathrm{~mm}$. This was a significant improvement compared with the performance prior to renewal. It is attributable to an improvement in the trackbed and sleeper support conditions, as the underlying embankment did not feature in the remedial works. 

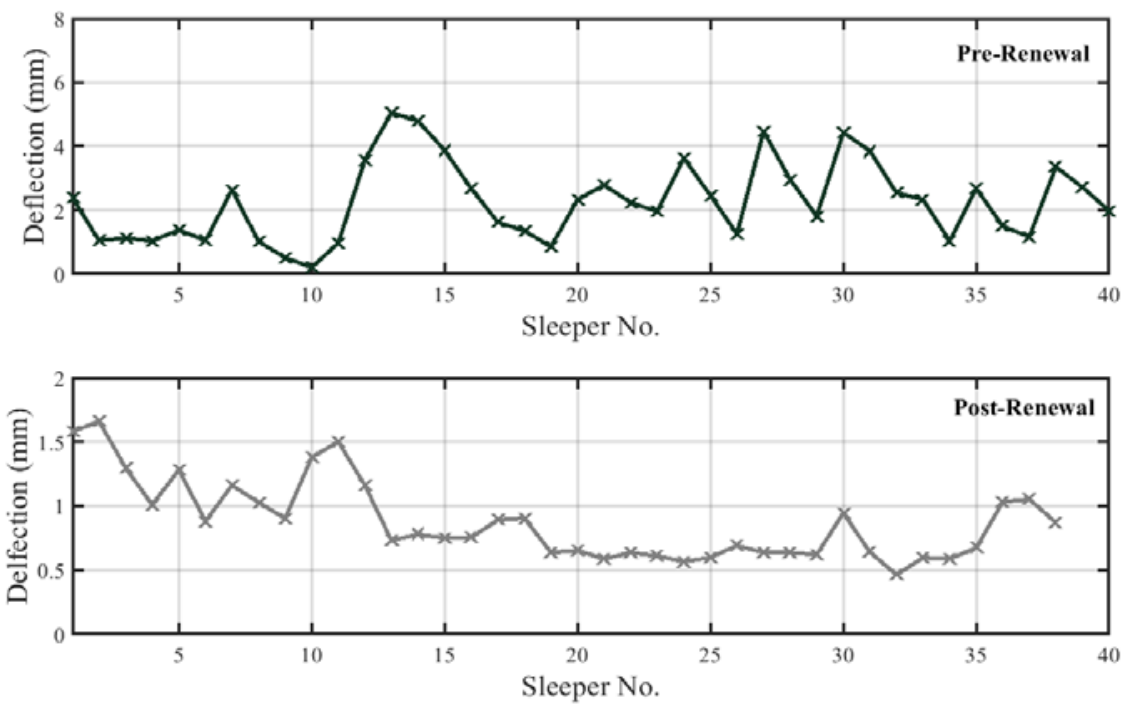

Fig. 5. Variation of deflection and system support modulus before and after a renewal.

\section{$4 \quad$ Summary}

Evidence based large scale and long-term studies of railway track infrastructure performance will require monitoring at an increased scale. Doing this from the infrastructure itself will necessitate the use of much lower cost monitoring equipment, and automation of data processing and interpretation. Low cost MEMS accelerometers, read by microcontrollers, are suitably low noise and have the appropriate range, resolution and bandwidth to evaluate track deflection and stiffness from data of vibrations induced by passing trains. Using data from this type of device, this paper has demonstrated how existing (and automatable) techniques can be used to classify and interpret track vibration signals from larger scale deployments to study variations in track performance along a railway. Interpretation of the results from this type of analysis is intuitive; the performance and condition of the track are clear, and the effects of interventions can be evaluated directly.

\section{$5 \quad$ Acknowledgements}

The research was funded by the Engineering and Physical Sciences Research Council (EPSRC) through the program grant Track to the Future (EP/M025276/1). This work would also not have been possible without the kind assistance given by staff from Network Rail and Network Rail High Speed and members of the infrastructure research group at the University of Southampton. 


\section{References}

1. British Standards Institution, BS EN 13848. Railway applications - Track Track geometry quality, in Part 2:Measuring systems - Track recording vehicles. 2006: London.

2. Auersch, L., The excitation of ground vibration by rail traffic: theory of vehicle-track-soil interaction and measurements on high-speed lines. Journal of Sound and Vibration, 2005. 284.1-2: 103-132 DOI: http://dx.doi.org/10.1016/j.jsv.2004.06.017.

3. Cui, Y.-J., et al., Investigation of interlayer soil behaviour by field monitoring. Transportation Geotechnics, 2014. 1.3: 91-105 DOI: http://dx.doi.org/10.1016/j.trgeo.2014.04.002.

4. Le Pen, L., et al., Behaviour of under sleeper pads at switches and crossings Field measurements. Proceedings of the Institution of Mechanical Engineers, Part F: Journal of Rail and Rapid Transit, 2017. 232.4: 1049-1063 DOI: 10.1177/0954409717707400.

5. Mishra, D., et al., An integrated approach to dynamic analysis of railroad track transitions behavior. Transportation Geotechnics, 2014. 1.4: 188-200 DOI: http://dx.doi.org/10.1016/j.trgeo.2014.07.001.

6. Paixão, A., E. Fortunato, and R. Calçada, Transition zones to railway bridges: Track measurements and numerical modelling. Engineering Structures, 2014. 80. 435-443 DOI: https://doi.org/10.1016/j.engstruct.2014.09.024.

7. Bowness, D., et al., Monitoring the dynamic displacements of railway track. Proceedings of the Institution of Mechanical Engineers, Part F: Journal of Rail and Rapid Transit, 2007. 221.1: 13-22 DOI: 10.1243/0954409jrrt51.

8. Thompson, D., Railway noise and vibration mechanisms, modelling and means of control. 2009, Elsevier: Oxford, UK. p. xv, 518 p.

9. Oppenheim, A.V. and R.W. Schafer, Digital signal processing. 1975: Prentice-Hall.

10. Milne, D., et al., Proving MEMS Technologies for Smarter Railway Infrastructure. Procedia Engineering, 2016. 143. 1077-1084 DOI: http://dx.doi.org/10.1016/j.proeng.2016.06.222.

11. Milne, D., et al., Automated processing of railway track deflection signals obtained from velocity and acceleration measurements. Proceedings of the Institution of Mechanical Engineers, Part F: Journal of Rail and Rapid Transit, 2018.

12. Powrie, W., et al., Train loading effects in railway geotechnical engineering: Ground response, analysis, measurement and interpretation. Transportation Geotechnics, 2019. 21. $100261 \quad$ DOI: https://doi.org/10.1016/j.trgeo.2019.100261.

13. Priest, J. and W. Powrie, Determination of Dynamic Track Modulus from Measurement of Track Velocity during Train Passage. Journal of Geotechnical and Geoenvironmental Engineering, 2009. 135.11: 1732-1740 DOI: doi:10.1061/(ASCE)GT.1943-5606.0000130. 
14. Timoshenko, S. and B.F. Langer, Stresses in railroad track. ASME Transactions, 1932. 54. 277-293.

15. Esveld, C., Modern railway track. Second Edition ed. 2001, Delft: MRTProductions.

16. Le Pen, L., et al., Evaluating railway track support stiffness from trackside measurements in the absence of wheel load data. Canadian Geotechnical Journal, 2016. 53.7: 1156-1166 DOI: 10.1139/cgj-2015-0268.

17. Ju, S.-H., H.-T. Lin, and J.-Y. Huang, Dominant frequencies of train-induced vibrations. Journal of Sound and Vibration, 2009. 319.1-2: 247-259 DOI: http://dx.doi.org/10.1016/j.jsv.2008.05.029.

18. Milne, D., et al., Properties of train load frequencies and their applications. Journal of Sound and Vibration, 2017. 397. 123-140 DOI: https://doi.org/10.1016/j.jsv.2017.03.006.

19. Le Pen, L., et al., A model for the stochastic prediction of track support stiffness. Proceedings of the Institution of Mechanical Engineers, Part F: Journal of Rail and Rapid Transit, 2019 DOI: 10.1177/0954409719841800.

20. Milne, D., et al., The influence of variation in track level and support system stiffness over longer lengths of track for track performance and vehicle track interaction. Vehicle System Dynamics, 2019: 1-24 DOI: 10.1080/00423114.2019.1677920. 